03,12

\title{
Экситоны и биэкситоны в сфероидальных квантовых точках $\mathrm{A}_{2} \mathrm{~B}_{6}$
}

\author{
(C) А.А. Головатенко, М.А. Семина, А.В. Родина, Т.В. Шубина \\ Физико-технический институт им. А.Ф. Иофрфе РАН, \\ Санкт-Петербург, Россия \\ E-mail: sasha.pti@mail.ioffe.ru
}

\begin{abstract}
В пределе сильного размерного квантования рассмотрены нижние энергетические состояния экситонов и биэкситонов в сфероидальных квантовых точках полупроводников с четырехкратно вырожденной вершиной валентной зоны, являющиеся активными в дипольном приближении при одно- и двухфотонном возбуждении. Выполнен сравнительный анализ порядка уровней размерного квантования дырки в потенциалах бесконечно глубокой квантовой ямы и трехмерного гармонического осциллятора с учетом аксиальной анизотропии формы квантовой точки (КТ). Показано, что анизотропия формы КТ может приводить к противоположному знаку расщепления по проекции углового момента $\pm 3 / 2, \pm 1 / 2$ для пространственно-нечетных $\left(1 P_{3 / 2}\right)$ и четных $\left(1 S_{3 / 2}\right)$ уровней дырки. При этом в случае потенциала бесконечно глубокой квантовой ямы может наблюдаться инверсия порядка $1 S_{3 / 2}$ и $1 P_{3 / 2}$ уровней при значениях отношения эффективных масс легкой и тяжелой дырки $\beta=m_{\mathrm{lh}} / m_{\mathrm{hh}} \approx 0.14$. Предложен вид пробных волновых функций дырки для состояния $1 P_{3 / 2}$ в потенциале изотропного трехмерного гармонического осциллятора в зависимости от $\beta$. Приведена зависимость энергии связи экситонов в рассматриваемых потенциалах от $\beta$ и рассмотрена возможность формирования различных биэкситонных состояний.
\end{abstract}

Работа выполнена при поддержке Российского научного фонда (проект № 14-22-00107).

DOI: $10.21883 /$ FTT.2018.08.46236.02Gr

\section{1. Введение}

Обычно при изучении краевой фотолюминесценции в квантовых точках (КТ) соединений $\mathrm{A}_{2} \mathrm{~B}_{6}$ интересуются основным экситонным состоянием $1 S_{e} 1 S_{3 / 2}$ $\left(1 S_{e}, 1 S_{3 / 2}\right.$ - основные уровни энергии электрона и дырки, соответственно), отвечающим за однофотонные процессы [1]. Недавно было показано [2], что наличие заряженных дефектов на поверхности КТ может подмешивать к основному состоянию экситона ближайший по энергии экситон $1 S_{e} 1 P_{3 / 2}$, отвечающий за край двухфотонного поглощения. Это делает подобные КТ интересным объектом для изучения процессов генерации второй оптической гармоники [3]. Кроме того, учет состояния дырки $1 P_{3 / 2}$ представляет дополнительный интерес при изучении биэкситонов. При малой разнице между энергиями уровней дырки $1 P_{3 / 2}$ и $1 S_{3 / 2}$ можно ожидать возникновения дополнительных биэкситонных состояний $1 S_{e}^{2} 1 S_{3 / 2}^{1} P_{3 / 2}^{1}$ с различным значением углового момента вблизи основного состояния биэкситона $1 S_{e}^{2} 1 S_{3 / 2}^{2}$.

Как эффективность подмешивания, так и положение биэкситонных уровней зависят от разницы энергий уровней дырки $1 P_{3 / 2}$ и $1 S_{3 / 2}$, определяемой видом локализующего потенциала и параметрами Латтинжера конкретного полупроводника. Квантование дырок в КТ, получаемых методом коллоидного синтеза, может быть с хорошей точностью описано потенциалом $V_{\text {box }}(r)$ сферически симметричной квантовой ямы радиуса $a$ с бесконечными барьерами при $r>a$ [4]. В случае же новых КТ $\mathrm{CdSe} / \mathrm{ZnSe}$, получаемых методом молекулярно-лучевой эпитаксии [5], более уместно рассматривать плавный потенциал, отражающий градиентное изменение состава между КТ и матрицей.
В настоящей работе мы приводим результаты расчета уровней энергии для состояний дырки $1 S_{3 / 2}$ и $1 P_{3 / 2}$ в потенциале $\quad V_{\mathrm{box}}(r)$ и потенциале сферически-симметричного гармонического осциллятоpa $V_{\text {osc }}(r)=k r^{2} / 2$ (где $k-$ жесткость потенциала) в зависимости от отношения масс легкой и тяжелой дырок $\beta=m_{\mathrm{lh}} / m_{\mathrm{hh}}$. Произведен анализ анизотропного расщепления уровней дырки $1 S_{3 / 2}$ и $1 P_{3 / 2}$ и найдены энергии связи $1 S_{e} 1 S_{3 / 2}$ и $1 S_{e} 1 P_{3 / 2}$ экситонов в зависимости от $\beta$ в потенциалах $V_{\mathrm{box}}(r)$ и $V_{\mathrm{osc}}(r)$.

\section{2. Решение задачи для сферически-симметричного потенциала}

Состояния дырок в сферически симметричном потенциале $V(r)$ характеризуются значениями полного момента $\mathbf{J}=\mathbf{l}+\mathbf{j}$ и вырождены по значениям его проекции М на произвольно выбранное направление наблюдения [4,6]. Здесь j и I операторы внутреннего $(j=3 / 2)$ и внешнего орбитального момента, соответственно. Нижние уровни размерного квантования соответствуют $J=3 / 2$ и делятся на пространственно-четные $1 S_{3 / 2}(l=0,2)$ и нечетные $1 P_{3 / 2}(l=1,3)$ состояния, энергии которых могут быть найдены в результате решения уравнения Шредингера

$$
\hat{H}_{L} \Phi_{J, M}(r)+V(r) \Phi_{J, M}(r)=E_{J, M} \Phi_{J, M}(r),
$$

где $\hat{H}_{L}$ - гамильтониан Латтинжера в сферическом приближении. Задача сводится к решению систем дифференциальных уравнений для радиальных функций $R_{0}(r)$ 
и $R_{2}(r)$ состояния $1 S_{3 / 2}$ и функций $R_{1}(r)$ и $R_{3}(r)$ состояния $1 P_{3 / 2}[6]$. В случае потенциала $V_{\text {box }}(r)$ уравнение (1) может быть решено точно для произвольного значения отношения масс легкой и тяжелой дырок $\beta=m_{\mathrm{lh}} / m_{\mathrm{hh}}$, a волновые функции дырки $R_{l}(r)$ выражаются через сферические функции Бесселя $[2,7]$. Зависимости энергий квантования от $\beta$ для состояний $1 S_{3 / 2}$ и $1 P_{3 / 2}$ приведены на рис. $1, a$.

В случае потенциала $V_{\text {osc }}(r)$ мы решаем задачу, следуя [8], как численным, так и вариационным методом. Способ построения пробных функций основывается на нахождении вида $R_{l}(r)$ при предельных значениях $\beta=0$ и $\beta=1$. При $\beta=1$ волновые функции $R_{0}(r)$ и $R_{1}(r)$ имеют тот же вид, что и функции для $1 s$ и $1 p$ уровней трехмерного гармонического осциллятора в невырожденной зоне [9]. При $\beta=0$ функции $R_{l}(r)$ и $R_{l+2}(r)$ должны удовлетворять дополнительным дифференциальным уравнениям, приведенным в [10]. Данные условия могут быть выполнены, если взять функции $R_{0}(r)$ и $R_{2}(r)$ в виде функций $2 s$ и $1 d$, а для $R_{1}(r)$ и $R_{3}(r)$ в виде функций $2 p$ и $1 f$ состояний осциллятора, соответственно [9]. Для конечной величины $\beta$ выбранные пробные функции должны плавно интерполировать между предельными формами. Функции $R_{0}(r)$ и $R_{2}(r)$ состояния $1 S_{3 / 2}$ даны в [8], а для состояния $1 P_{3 / 2}$ пробные радиальные волновые функции имеют следующий вид:

$$
\begin{gathered}
R_{1}(r)=7.5 C / L^{3 / 2}\left(\alpha_{0}\right)^{1 / 2} \tilde{r}\left[\exp \left(-\alpha_{0} \tilde{r}^{2}\right)\right. \\
\left.+\alpha_{1} \exp \left(-\alpha_{0} \beta^{0.25} \tilde{r}^{2}\right)\right]-3 R_{3}(r), \\
R_{3}(r)=C / L^{3 / 2}\left(\alpha_{0}\right)^{3 / 2} \tilde{r}^{3}\left[\exp \left(-\alpha_{0} \tilde{r}^{2}\right)\right. \\
\left.-\alpha_{2} \exp \left(-\alpha_{0} \beta^{0.25} \tilde{r}^{2}\right)\right],
\end{gathered}
$$

где $C-$ нормировочная константа, $\alpha_{i}-$ вариационные параметры, $\tilde{r}=r / L, L=\left(\hbar^{2} / m_{\mathrm{hh}} k\right)^{1 / 4}-$ характерная длина потенциала $V_{\text {osc }}(r)$. Пробные функции дают хорошее согласие с численным расчетом для зависимости энергий квантования от $\beta$ (рис. $1, a$ ). Можно видеть, что в обоих потенциалах существуют значения $\beta$, при которых разница энергий уровней $1 S_{3 / 2}$ и $1 P_{3 / 2}$ достигает минимума.

\section{3. Анизотропное расщепление}

Аксиальная анизотропия КТ, имеющих форму эллипсоида вращения с полуосями $b$ и $c$, приводит к расщеплению уровней энергии дырки с проекцией момента $M= \pm 3 / 2$ и $M= \pm 1 / 2$. При малых значениях параметра анизотропии $\mu=c / b-1$ уровни энергии могут быть найдены в рамках первого порядка теории возмущений $[8,11]$ как $E_{ \pm 1 / 2}(\beta)=E(\beta)[1+\mu u(\beta)]$ и $E_{ \pm 3 / 2}(\beta)=E(\beta)[1-\mu u(\beta)]$. Здесь $u(\beta)-$ безразмерная функция, выраженная через найденные радиальные функции $R_{0}(r)$ и $R_{2}(r)$ для $1 S_{3 / 2}$ (см. [8]) и $R_{1}(r)$ и $R_{3}(r)$ для $1 P_{3 / 2}$ состояний, соответственно. Отметим, что задача для потенциала анизотропного гармонического


Рис. 1. Зависимость от $\beta$ для: (a) энергии размерного квантования дырки $E(\beta)$ в единицах $\hbar^{2} \pi^{2} / 2 m_{\mathrm{hh}} a^{2}$, где $a-$ радиус КТ для $V_{\mathrm{box}}(r)$, и $a=L / \sqrt{3}$ для потенциала $V_{\mathrm{osc}}(r)$ с характерной длиной $L=\left(\hbar^{2} / m_{\mathrm{hh}} k\right)^{1 / 4} ; \quad(b)$ безразмерной функции анизотропного расщепления $u(\beta)=\left[E_{ \pm 1 / 2}(\beta)-E_{ \pm 3 / 2}(\beta)\right] / 2 \mu E(\beta)$.

осциллятора может быть напрямую решена численным методом $[8,12]$, что позволяет получить оценку области применимости теории возмущений. Безразмерные функции $u(\beta)$ для состояний $1 S_{3 / 2}$ и $1 P_{3 / 2}$ в рассматриваемых потенциалах, приведены на рис. $1, b$. Важно отметить, что функции $u(\beta)$ имеют разный знак для всех значений $\beta$ в гармоническом потенциале и в некоторой области значений $\beta$ в потенциале $V_{\mathrm{box}}(r)$. Результирующие уровни энергии в вытянутых КТ с $\mu=0.2$ приведены на pис. 2, $a$ и $b$. Для потенциала $V_{\text {box }}(r)$ область значений $\beta$, где наблюдается максимальное сближение уровней $1 S_{3 / 2}$ и $1 P_{3 / 2}$, совпадает с областью максимума для функции расщепления состояния $1 P_{3 / 2}$ и минимума для функции расщепления $1 S_{3 / 2}$. В результате происходит инверсия порядка уровней, и состояние $1 P_{3 / 2}$ становится основным (рис. 2,a). Для КТ с гармоническим потенциалом инверсии порядка уровней не происходит (рис. 2, $b$ ). 
$a$
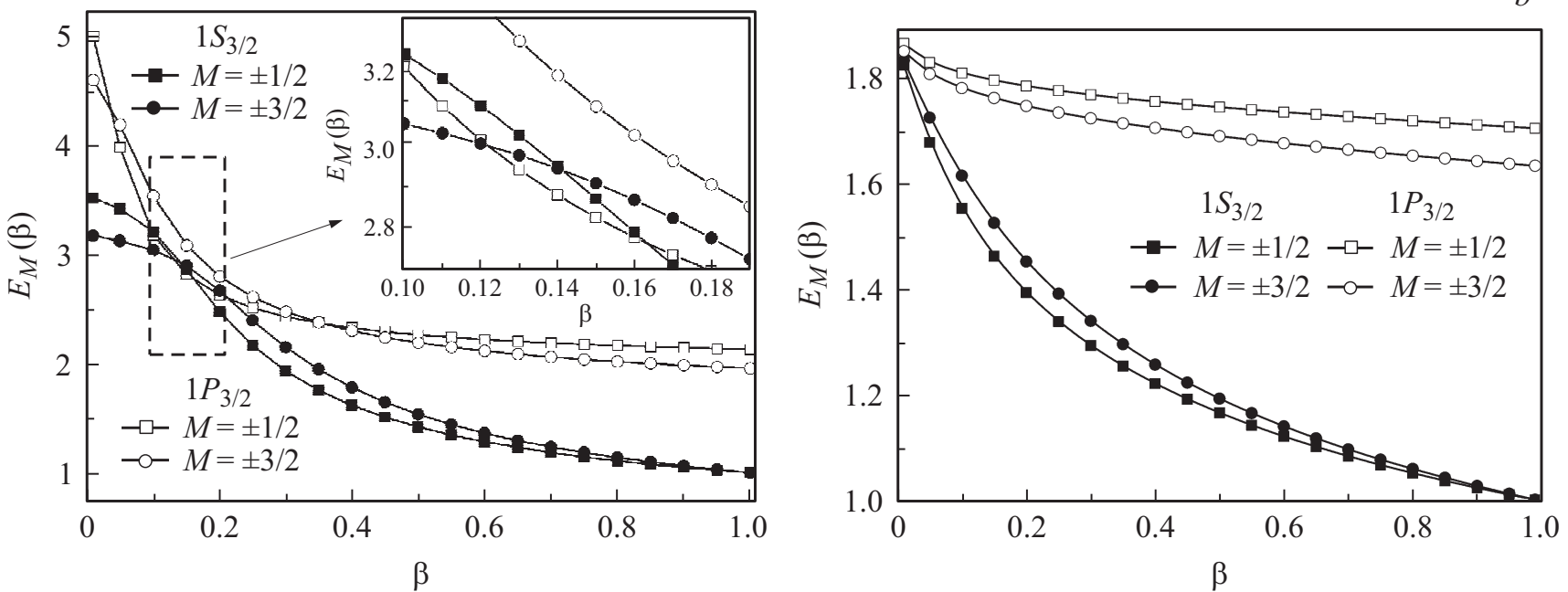

Рис. 2. Зависимость от $\beta$ положения уровней энергии дырки $E_{M}(\beta)$ с различной проекцией полного момента $|M|$ на ось анизотропии при параметре анизотропии $\mu=0.2$ для: $a-$ потенциала $V_{\mathrm{box}}(r), b-$ потенциала $V_{\text {osc }}(r)$. Единицы энергии те же, что и на рис. $1, a$.

\section{4. Экситоны и биэкситоны}

Используя найденные волновые функции дырки и известные волновые функции $1 S_{e}$ уровня электрона [13], можно найти величину энергии связи экситона $E_{x}^{b}$ в рассматриваемых потенциалах. Если энергия квантования носителей достаточно велика, то корреляцией между состояниями электрона и дырки можно пренебречь и использовать теорию возмущений первого порядка для оценки $E_{x}^{b}$. Результаты расчета энергии связи для $1 S_{e} 1 S_{3 / 2}$ и $1 S_{e} 1 P_{3 / 2}$ экситонов представлены на рис. 3.

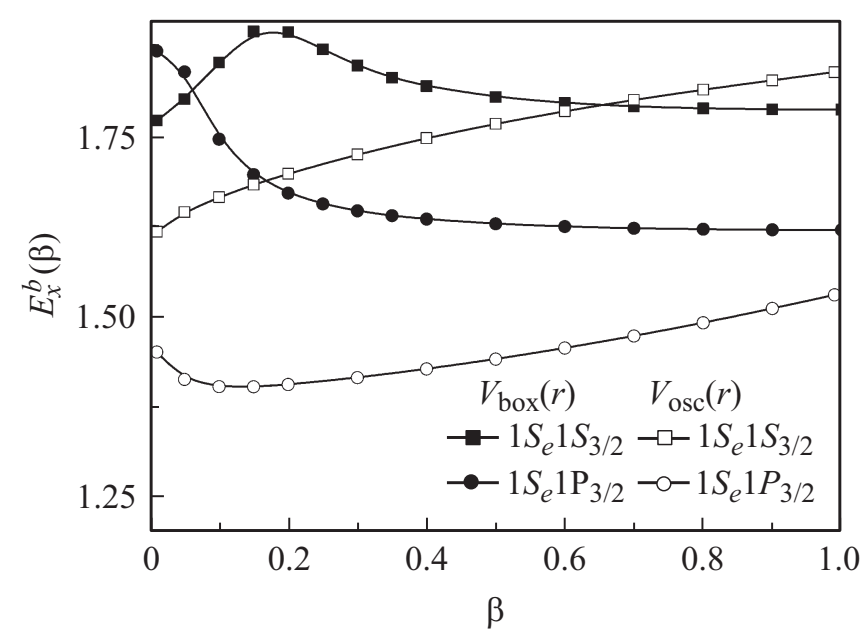

Рис. 3. Зависимость энергии связи экситона от $\beta$ для экситонов $1 S_{e} 1 S_{3 / 2}$ и $1 S_{e} 1 P_{3 / 2}$ в КТ с потенциалом $V_{\text {box }}(r)$ и $V_{\text {osc }}(r)$. Параметры потенциалов $V_{\text {osc }}(r)$ и $V_{\text {box }}(r)$ выбраны таким образом, что средние значения координат для электронов и дырок равны при $\beta=1$. Единицы энергии: $E^{2} / \varepsilon a, \varepsilon-$ диэлектрическая проницаемость, $a-$ радиус КТ для $V_{\mathrm{box}}(r)$ и $a=L / \sqrt{3}$ для потенциала $V_{\mathrm{osc}}(r)$.
Параметры потенциалов выбраны таким образом, чтобы при $\beta=1$ среднее значение координаты $\bar{r}_{e, h}$ электрона и дырки были равны для обоих потенциалов. С уменьшением $\beta$ величина $\bar{r}_{h}$ в потенциале $V_{\text {osc }}(r)$ возрастает, a кулоновское притяжение падает в силу уменьшения интеграла перекрытия волновых функций электрона и дырки. В случае потенциала $V_{\mathrm{box}}(r)$ из-за ограничения $\bar{r}_{h}<a$ происходит перестройка волновой функций дырки, что приводит к немонотонной зависимости от $\beta$ энергии связи для $1 S_{e} 1 S_{3 / 2}$ экситона.

Для расчета энергии биэкситонов, состоящих из двух электронов $1 S_{e}$ и двух дырок с рассмотренных уровней размерного квантования $J=3 / 2\left(1 S_{e}^{2} 1 S_{3 / 2}^{2}, 1 S_{e}^{2} 1 P_{3 / 2}^{2}\right.$, $\left.1 S_{e}^{2} 1 S_{3 / 2}^{1} 1 P_{3 / 2}^{1}\right)$ необходимо учитывать корреляции между состояниями электронов и дырок, а также энергию обменного взаимодействия между дырками, зависящую от их суммарного углового момента. Это может быть сделано вариационным методом с использованием пробных волновых функций, представленных в данной работе, а также в работах $[8,13]$. При этом кулоновское взаимодействие между двумя дырками приводит к смешиванию $1 S_{e}^{2} 1 S_{3 / 2}^{2}$ и $1 S_{e}^{2} 1 P_{3 / 2}^{2}$ биэкситонных состояний.

\section{5. Заключение}

Нами впервые предложены пробные волновые функции для состояния дырки $1 P_{3 / 2}$ в потенциале сферически-симметричного гармонического осциллятоpa для конечного отношения масс легкой и тяжелой дырки. Пробные волновые функции для состояния $1 P_{3 / 2}$ и ранее найденные нами пробные волновые функции для состояния $1 S_{3 / 2}$ [8] дают хорошее согласие с численным расчетом как для энергии квантования дырки, так и для величины анизотропного расщепления. Эти функции 
могут быть использованы для описания оптических переходов с участием одного и двух фотонов, а также для вариационного расчета энергии связи биэкситонов с различной конфигурацией дырочных состояний. Отметим, что пробные функции, предложенные для КТ с гармоническим потенциалом, могут быть также использованы в случае плавного потенциала с конечной высотой барьера [8], отражающего разрыв зон в реальных эпитаксиальных структурах. Анизотропное расщепление состояний дырки с различным модулем полного углового момента $M$ в гармоническом потенциале имеет противоположный знак для уровней $1 S_{3 / 2}$ и $1 P_{3 / 2}$ при любом значении $\beta$. Для потенциала бесконечно глубокой квантовой ямы анизотропного расщепление меняет знак при различных $\beta$ для уровней $1 S_{3 / 2}$ и $1 P_{3 / 2}$. В сфероидальных КТ со степенью анизотропии $|\mu| \geq 0.1$ происходит инверсия порядка уровней дырки $1 S_{3 / 2}$ и $1 P_{3 / 2}$ в области $\beta \approx 0.14$. Можно предположить, что в таких КТ с резким потенциальным барьером может наблюдаться пик двухфотонного поглощения, соответствующий экситону с дыркой в состоянии $1 P_{3 / 2}$, на энергии приблизительно равной половине энергии поглощения оптически активного экситона, образованного дыркой в состоянии $1 S_{3 / 2}$.

\section{Список литературы}

[1] Al.L. Efros, M. Rosen, M. Kuno, M. Nirmal, D.J. Norris, M. Bawendi. Phys. Rev. B 54, 4843 (1996).

[2] P.C. Sercel, A. Shabaev, A1.L. Efros. Nano Lett. 17, 4820 (2017).

[3] M. Lafrentz, D. Brunne, B. Kaminski, V.V. Pavlov, A.V. Rodina, R.V. Pisarev, D.R. Yakovlev, A. Bakin, M. Bayer. Phys. Rev. Lett. 110, 116402 (2013).

[4] A.I. Ekimov, F. Hache, M.C. Schanne-Klein, D. Ricard, C. Flytzanis, I.A. Kudryavtsev, T.V. Yazeva, A.V. Rodina, A1.L. Efros. J. Opt. Soc. Am. B 10, 100 (1993).

[5] T.V. Shubina, A.V. Rodina, M.A. Semina, A.A. Golovatenko, A.A. Toropov, M.V. Rakhlin, I.V. Sedova, S.V. Sorokin, S.V. Gronin, A.A. Sitnikova, D.I. Kuritsyn, S.M. Sergeev, Z.F. Krasil'nik, S.V. Ivanov. Phys. Status Solidi 253, 1485 (2016).

[6] Б.Л. Гельмонт, М.И. Дьяконов. ФТП 5, 2191 (1971).

[7] Г.Б. Григорян, Э.М. Казарян, Ал.Л. Эфрос, Т.В. Язева. ФТТ 32, 1772 (1990).

[8] M.A. Semina, A.A. Golovatenko, A.V. Rodina. Phys. Rev. B 93, 045409 (2016).

[9] А.С. Давыдов. Квантовая механика. Наука, М. (1973). $704 \mathrm{c}$.

[10] A.A. Golovatenko, M.A. Semina, A.V. Rodina, T.V. Shubina. In: Book of abstracts $25^{\text {th }}$ Int. Symp. Nanostructures: Physics and Technology, Saint-Petersburg (2017). P. 206.

[11] Al.L. Efros, A.V. Rodina. Phys. Rev. B 47, 10005 (1993).

[12] М.A. Семина, Р.А. Сурис. ФТП 49, 817 (2015).

[13] А.А. Головатенко, М.А. Семина, А.В. Родина, Т.В. Шубина. ФTT 59, 1192 (2017).

Редактор Ю.Э. Китаев 